\title{
Comparison of moderate and advanced glaucoma patients in Ghana
}

This article was published in the following Dove Press journal:

Clinical Ophthalmology

23 February 2012

Number of times this article has been viewed

\author{
Andrew W Francis' \\ Michael E Gyasi² \\ Li Deng ${ }^{3}$ \\ Haiyan Gong' \\ 'Boston University School of \\ Medicine, Boston, MA, USA; \\ ${ }^{2}$ Emmanuel Eye Centre, Accra, Ghana; \\ ${ }^{3}$ The New England College \\ of Optometry, Boston, MA, USA
}

\begin{abstract}
Purpose: To compare moderate and advanced glaucoma patients in Ghana.
Methods: A retrospective cross-sectional study of 164 patients with primary open-angle glaucoma (POAG) were separated into moderate and advanced glaucoma groups. Definitions of moderate and advanced POAG were derived from International Geographical and Epidemiologic Ophthalmology criteria and included clinical assessment of optic disc atrophy and Humphrey automated perimetry. Data were collected at the patient's first visit prior to initiation of therapy. Eligible POAG patients included those $\geq 30$ years old with reliable Humphrey visual field (HVF) results, no past POAG diagnosis, treatment, or evidence of a secondary cause for glaucoma. Main outcome measures included comparisons of intraocular pressure (IOP), cup-to-disk ratio (CDR), best corrected visual acuity (VA), age, Humphrey mean deviation (MD), and pattern standard deviation (PSD).

Results: Of 164 charts reviewed, 90 (54.9\%) advanced and 74 (45.1\%) moderate POAG patients were compared. Mean age was 59.36 versus 55.53 years, respectively. Significant differences in IOP, CDR, CDR asymmetry, and HVF results were described. IOP $>30 \mathrm{mmHg}$ was associated with $\mathrm{CDR}>0.7$ and MD greater than $-12 \mathrm{~dB}$ in both eyes.

Conclusion: Significant differences were found between IOP, CDR, MD and PSD values. HVF is predictive of pretreated IOP, CDR, and severity of POAG and it is strongly encouraged as part of the standard glaucoma work up in all Ghanaian patients.
\end{abstract}

Keywords: glaucoma, intraocular pressure, Ghana

\section{Introduction}

Primary open-angle glaucoma (POAG) is the leading cause of irreversible blindness worldwide. ${ }^{1}$ The number of persons affected is estimated to be in the region of 66.8 million with 6.7 million blind. ${ }^{2}$ This disorder is characterized by progressive optic neuropathy eventually leading to loss of central vision and total blindness in its final stages. A population study in Ghana reported that POAG affects $7.7 \%$ and $8.5 \%$ of people 30 and 40 years of age, respectively ${ }^{3}$ whereas $1.9 \%$ of Americans over 40 years of age are reported affected. ${ }^{4}$

Established risk factors for POAG in Ghanaian patients include elevated intraocular pressure (IOP), a family history, old age, and African ancestry. ${ }^{3}$ Recent studies in Ghana have also identified late presentation and a delay in diagnosis as significant risk factors. ${ }^{5,6}$ Treatment consists of lowering the IOP through medical or surgical methods. ${ }^{7}$ As of 2004, there are 45 ophthalmologists practicing in Ghana, or approximately one per 500,000 persons. $^{8}$ The majority of these physicians practice in the outpatient setting near the capital city of Accra. Patients in this practice setting are the focus of this manuscript.
Correspondence: Andrew W Francis Boston University School of Medicine, 72 East Concord Street, 9th floor, Boston, MA 02118, USA

Tel + I 5 I0 2928058

Fax +I 6176385337

Email andrew12@bu.edu 
This study aims to describe and compare two POAG patient groups at separate stages of disease severity for associations between clinical findings including IOP, cup-to-disk ratio (CDR), CDR asymmetry, age, visual acuity (VA) and Humphrey visual field (HVF) mean deviation (MD), and pattern standard deviation (PSD).

\section{Methods}

This was a retrospective cross-sectional study involving 164 patient charts reviewed at the Emmanuel Eye Clinic, a large outpatient eye clinic in Accra, Ghana with an annual volume that exceeds 30,000 patients. Data were gathered from the patients' initial visits prior to initiation of medical therapy.

\section{Inclusion criteria}

Two broad patient groups were formed and defined according to modified International Geographical and Epidemiologic Ophthalmology (ISGEO) POAG criteria (Table 1). ${ }^{9}$ The first group was defined as having moderate glaucoma, with clinical findings showing disk cupping (CDR 0.7-0.8) without a visual field defect (VFD) or a CDR $<0.7$ with a confirmed VFD. The second group was defined as having advanced glaucoma, with clinical findings showing either advanced structural and functional damage (CDR $>0.7$ with a confirmed VFD) or advanced optic nerve atrophy (CDR $>0.9)$ with or without satisfactory HVF results. Eligible patients included those $\geq 30$ years old with a diagnosis of POAG on record, no past diagnosis or treatment for glaucoma or ocular hypertension elsewhere and no history or clinical evidence for a secondary or congenital cause of glaucoma.

Table I Definitions of moderate and advanced primary openangle glaucoma used in this study

\section{Advanced POAG group}

Category I: a definite and reliable glaucomatous visual field defect was found in the presence of either $C D R \geq 0.7$ or a CDR asymmetry $\geq 0.2$ between adjacent eyes

Category 2: Humphrey 24-2 visual field testing was not completed satisfactorily, but the CDR was $\geq 0.9$, or CDR asymmetry was $\geq 0.3$

Moderate POAG group

CDR 0.7 or 0.8 without VFD; category I, but not category 2, optic disc criteria met, but were not proven to have reliable or definite visual field defects

CDR $<0.7$ with a definitive VFD; Those with definitive visual field defects, but not meeting category I disc criteria or those with optic disc margin hemorrhages

Note: Advanced and moderate glaucoma definitions were modified from International Geographical and Epidemiologic Ophthalmology classifications first published by Foster P, et al. ${ }^{9}$

Abbreviations: POAG, primary open-angle glaucoma; CDR, cup-to-disk ratio; $\mathrm{VFD}$, visual field defect.
All patients had received at the initial visit a comprehensive slit-lamp examination, Goldmann applanation tonometry (Haag-Streit, Mason, OH), gonioscopy, and 24-2 HVF at their initial presentation. Only reliable HVF results were included in this study. We chose to base a patient's IOP on the average of two consecutive measurements taken from the patient chart prior to initiation of any medical therapy.

\section{Main outcome measures}

Patient groups were described and compared. Comparisons include age, gender, IOP, CDR, CDR asymmetry, HVF MD/PSD values and VA results. All VA data were recorded from a standard Snellen chart at 20 feet $(6 \mathrm{~m})$ and converted to $\log$ MAR format for standardized comparisons. Associations were derived using odds ratio (OR) comparisons. The moderate group functioned as the control.

\section{Definitions}

A VFD was determined by Humphrey computer-based automated perimetry, meaning positive results of hemimeridional analyses of threshold tests and/or the presence of one or more absolute defects in the central $30^{\circ}$ as tested with the full threshold strategy $24-2$ (central 54 points, two zone strategy) with ophthalmologic interpretation used for definitive diagnosis of glaucomatous field loss.

\section{Exclusion criteria}

Secondary or congenital causes of glaucoma were excluded from this study. Patients with prior trabeculectomy or medically controlled IOPs were excluded. A retinal disease that may cause visual field defects or macular disease which significantly impairs central vision was an exclusion criterion. Patients with closed angles on gonioscopy were diagnosed as angle closure glaucoma and excluded.

\section{Data analysis}

For one eye's data analysis (oculus dexter [OD] or oculus sinister [OS]), the two-sample $t$-test was applied to compare mean age, IOP, CDR, CDR asymmetry, MD/PSD and VA in $\log M A R$ between groups. Ordinal logistic regression was used in the comparison of VA when it was treated as ordinal data. For both eyes' data combined, the mixed model was used to account for between-eye correlations. The mixed model was also applied to assess correlations between MD and other risk factors when treating them as continuous variables. The associations of age $\geq 65$ years and IOP (worse IOP of two eyes $\geq 31 \mathrm{mmHg}$ ) with advanced glaucoma were assessed by calculating ORs via logistic 
regression models. Gender distribution difference between groups was done by the Chi-square test. The comparison of risk factors across groups defined by MD values was done using analysis of variance.

\section{Results}

Basic demographics of moderate and advanced POAG groups are shown in Table 2. Of 164 charts included, 90 (54.9\%) were advanced POAG patients and $74(45.1 \%)$ were moderate POAG patients. The mean age for all patients was 57.66 years with a standard deviation of 15.03 years. There was no significant difference in age between subject groups $(P=0.10)$. There was a highly significant difference in the gender distribution between the two groups $(P=0.0096)$. The proportion of males in the advanced group was higher than that in the moderate group ( $63.3 \%$ vs $40.5 \%$ ).

A higher percentage of patients in the advanced group were older than 65 years. All six patients older than 80 years of age had advanced POAG. The OR of having advanced POAG between patients older or younger than 65 years was $\mathrm{OR}=1.70(0.90-3.25)$, which is not statistically significant.

Clinical findings are summarized in Table 3. Advanced patients had significantly higher mean IOP on initial presentation in both eyes (OD: 30.93 vs $25.94 \mathrm{mmHg}, P<0.01$; OS: 32.37 vs $26.42 \mathrm{mmHg}, P<0.01$ ). The $\mathrm{OR}$ of being an advanced glaucoma patient with $\mathrm{IOP} \geq 31 \mathrm{mmHg}$ versus IOP $\leq 30 \mathrm{mmHg}$ was higher than for moderate patients (OR $2.24,95 \% \mathrm{CI} 1.17-4.29)$. Increasing IOP also correlated with increasing CDR (Figure 1). Nearly $95 \%$ of patients with an IOP $\geq 40 \mathrm{mmHg}$ had a CDR $\geq 0.7$. This correlation was significant for men and women in both eyes (OD $P=0.01$; OS $P<0.0001)$. Mean CDR was larger in advanced patients ( 0.85 vs $0.58, P<0.001)$ and mean CDR asymmetry was also greater $(0.14$ vs $0.05, P<0.001)$.

Table 2 Demographic characteristics of moderate and advanced primary open-angle glaucoma patients

\begin{tabular}{lll}
\hline Variable & Advanced patients & Moderate group \\
\hline Patients & 90 & 74 \\
Age years, mean (SD) & $59.36(14.8 \mathrm{I})$ & $55.53(15.13)$ \\
$\quad<35$ & 6 & 9 \\
$35-49$ & 22 & 13 \\
$50-64$ & 23 & 28 \\
$65-79$ & 34 & 23 \\
$\geq 80$ & 6 & 0 \\
Gender & & \\
$\quad$ Male & $57 / 90(63.3 \%)$ & $30 / 74(40.5 \%)$ \\
Female & $34 / 90(36.7 \%)$ & $43 / 74(59.5 \%)$ \\
\hline
\end{tabular}

Abbreviation: SD, standard deviation.
Table 3 Comparison of clinical findings between the moderate and advanced primary open-angle glaucoma patients

\begin{tabular}{llll}
\hline Variable & $\begin{array}{l}\text { Advanced } \\
\text { patients }\end{array}$ & $\begin{array}{l}\text { Moderate } \\
\text { group }\end{array}$ & P value \\
\hline $\begin{array}{l}\text { Patients } \\
\text { OD }\end{array}$ & 90 & 74 & \\
IOP mean (SD) & $30.93(\mathrm{I} .65)$ & $25.94(9.42)$ & $<0.0$ I \\
IOP $>30(\mathrm{no})$ & 35 & 18 & 0.08 \\
IOP range & $12-76$ & $10-60$ & \\
CDR & $0.84(0.16)$ & $0.57(0.14)$ & $<0.00$ I \\
OS & & & \\
IOP mean (SD) & $32.37(13.69)$ & $26.42(9.39)$ & $<0.01$ \\
IOP $>30(n o)$ & 39 & 20 & 0.05 \\
IOP range & $14-70$ & $10-65$ & \\
CDR & $0.85(0.16)$ & $0.58(0.14)$ & $<0.00$ I \\
CDR asymmetry & $0.14(0.16)$ & $0.05(0.07)$ & $<0.00$ I \\
\hline
\end{tabular}

Abbreviations: OD, oculus dexter; IOP, intraocular pressure; CDR, cup-to-disk ratio; OS, oculus sinister.

Advanced patients had poorer visual acuity in both eyes (Table 4). There was no significant difference in the number of patients with 20/20 correctable vision for either group, however, more advanced patients met or exceeded World Health Organization (WHO) criteria for legal blindness $(>20 / 200 \mathrm{VA})$ in at least one eye. When treating visual acuities measured from the Snellen chart as ordinal data $(20 / 20 \rightarrow$ NLP) a difference was found in both eyes (OD $P<0.05$; OS $P<0.05)$. Comparing VA converted to $\log$ MAR averages that excluded hand motion (HM), light perception (LP), and no light perception (NLP) for each eye showed a significant difference in OD $(P<0.05)$, but not in OS $(P=0.61)$. Poor vision was more often unilateral than bilateral: Less than $20 \%$ of advanced patients and only one moderate patient had VA $>20 / 200$ oculi uterque (OU).

HVF results are summarized in Table 5. The MD value in the advanced glaucoma group was significantly worse than in the moderate group $(-17.38 \mathrm{vs}-6.69 \mathrm{~dB}, P<0.001)$. The difference in PSD value between the moderate and advanced glaucoma group was not initially significant (6.71 vs $5.11 \mathrm{~dB}, P=0.18$ ) until adjusting for two outliers $(P<0.05)$. IOP in the advanced HVF group was significantly higher than the moderate HVF group (36.46 vs 23.03 $\mathrm{mmHg}, P<0.001)$. CDR was also significantly higher in the advanced group versus the moderate group $(0.83$ vs 0.57 , $P<0.001)$. Associations between HVF MD and PSD values with IOP, CDR, age, and VA are shown in Figures 2 and 3.

\section{Discussion}

This study compares two distinct primary open angle glaucoma patient groups defined according to clinical disease severity for important associations between common risk 
A

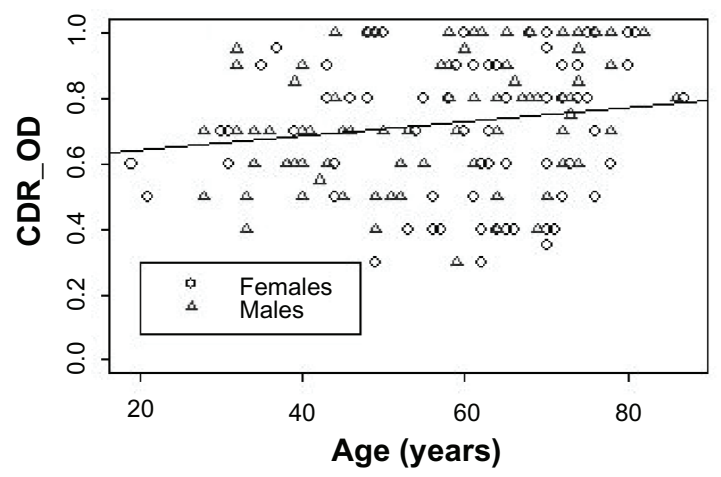

C

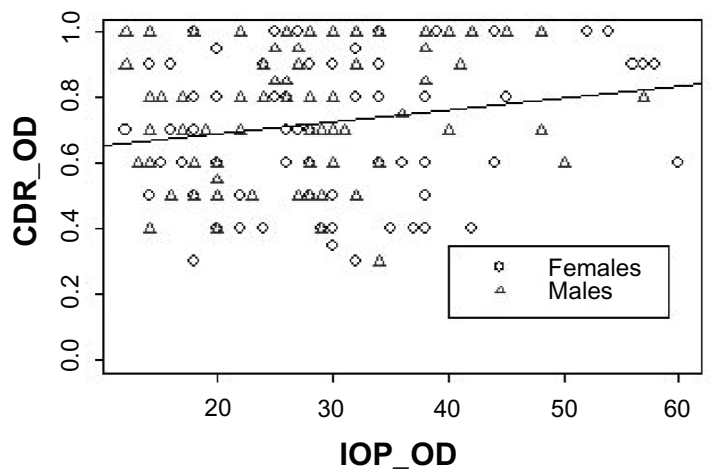

B

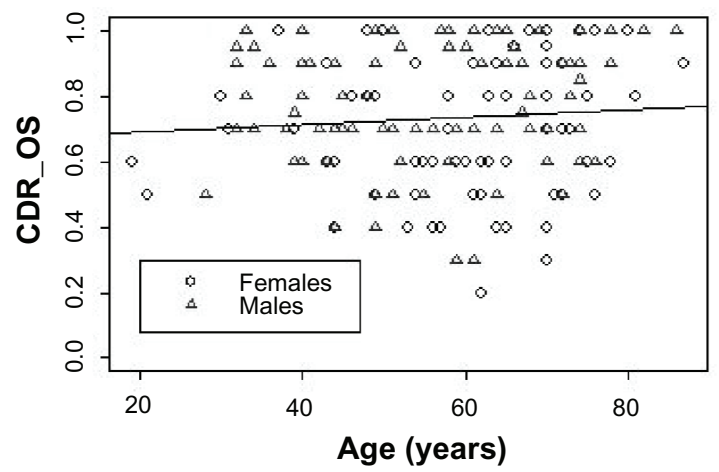

D

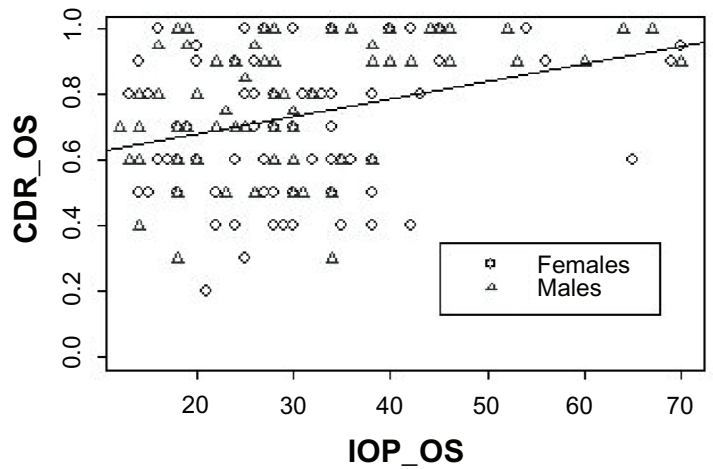

Figure I Comparisons of cup-to-disk ratio as a function of age and intraocular pressure for moderate and advanced primary open angle patient groups. Notes: Overall CDR is slightly positively correlated with age among advanced patients, but the correlation was significant only in $O D(P<0.05)$. CDR strongly correlated with IOP. (A) The relationship between CDR OD and age $(P<0.05)$. (B) CDR OS and age $(P=0.32)$. (C) CDR and IOP OD ( $P=0.0 \mathrm{I})$. (D) CDR and IOP OS ( $<<0.000 \mathrm{I})$. The linear relations between IOP/Age and CDR do not vary significantly by gender ( $P$ values for interaction between gender and slope $>0.05)$.

Abbreviations: CDR, cup-to-disk ratio; IOP, intraocular pressure; OD, oculus dexter; OS, oculus sinister.

factors. Patient groups were defined according to modified ISGEO criteria and included a relatively equal number with no significant differences in age or ancestry.

Herndon et al identified in a previous outpatient survey of 198 glaucoma patients in Ghana that the most common form of glaucoma was primary open-angle glaucoma (44.2\%) followed by POAG suspects (30.5\%) with chronic angle-closure glaucoma being rare $(6.6 \%) .{ }^{6}$ Our study sought to compare associations between moderate and advanced POAG patients that closely resembled POAG and POAG suspect groups in that study. In choosing to define one group as having moderate glaucoma versus glaucoma suspects, we took into account that all patients in our study were receiving active glaucoma therapy and were being treated clinically as a continuum from moderate to severe disease. It is possible that a small group of "nonprogressers" were included in the moderate glaucoma group. Even so, it is not possible to isolate a group of glaucoma suspects who are all nonprogressers either for comparison purposes with advanced patients. Using modified ISGEO classification is a reasonable alternative to define glaucoma patients with moderate stage disease at high risk for progression to advanced disease.

The mean age of glaucoma patients in our study was 57.66 years which is similar to the average age in many other studies carried out in African populations. ${ }^{10}$ These include studies in St Lucia, ${ }^{11}$ Barbados,${ }^{12}$ Tanzania, ${ }^{13}$ and South Africa. ${ }^{14}$ Direct comparisons with these population studies are difficult considering our smaller sample size and focus only on the outpatient clinical practice. While the vast majority of glaucoma patients that receive care do so in the outpatient setting, the vast majority of patients with glaucoma in Ghana are not receiving treatment so our findings cannot be applied to the population at large. We can conclude from our results that most cases of POAG at this outpatient site occur in patients older than 50 years of age.

Other studies, notably the early glaucoma manifest trial (EGMT), provide convincing evidence that POAG increases with age. ${ }^{15,16}$ The EGMT showed an increase in prevalence of POAG with age with less than $1 \%$ of patients $<65$ years 
Table 4 Comparison of best corrected visual acuities between moderate and advanced open-angle glaucoma patients

\begin{tabular}{|c|c|c|c|}
\hline Variable & $\begin{array}{l}\text { Advanced } \\
\text { patients }\end{array}$ & $\begin{array}{l}\text { Moderate } \\
\text { group }\end{array}$ & $P$ value \\
\hline Patients & 90 & 74 & \\
\hline \multicolumn{4}{|l|}{ OD } \\
\hline Categorical analysis & & & $<0.05$ \\
\hline $20 / 20$ & 18 & 15 & \\
\hline $20 / 25-20 / 60$ & 28 & 38 & \\
\hline $20 / 70-20 / 160$ & 13 & 5 & \\
\hline $20 / 200-20 / 2000$ & 7 & 5 & \\
\hline$>20 / 2000$ & 18 & 9 & \\
\hline $\mathrm{HM}$ & 3 & 1 & \\
\hline NLP & 4 & I & \\
\hline WHO blind & 32 & 16 & \\
\hline \multicolumn{4}{|l|}{ OS } \\
\hline Categorical analysis & & & $<0.05$ \\
\hline $20 / 20$ & 16 & 14 & \\
\hline $20 / 25-20 / 60$ & 35 & 40 & \\
\hline $20 / 70-20 / 160$ & 8 & 6 & \\
\hline $20 / 200-20 / 2000$ & 4 & 5 & \\
\hline$>20 / 2000$ & 11 & 8 & \\
\hline $\mathrm{HM}$ & 9 & 0 & \\
\hline NLP & 8 & 1 & \\
\hline WHO blind & 32 & 14 & \\
\hline \multicolumn{4}{|l|}{ OU } \\
\hline WHO blind OU & 15 & 1 & \\
\hline $\mathrm{HMOU}$ & 0 & 0 & \\
\hline NLP OU & 0 & 0 & \\
\hline
\end{tabular}

Notes: Comparisons of BCVA results showed advanced POAG patients had significantly poorer vision in OD $(P<0.05)$ and $O S(P<0.05)$. There was no significant difference in the number of patients with 20/20 correctable vision for either group, however, more advanced patients met or exceeded WHO criteria for legal blindness ( $>20 / 200$ BCVA) in at least one eye. Poor vision was more often unilateral than bilateral. Less than $20 \%$ of advanced patients and only one moderate patient had BCVA $>20 / 200$ OU.

Abbreviations: BCVA, best corrected visual acuity; HM, hand motion; NLP, no light perception; OD, oculus dexter; OS, oculus sinister; OU, oculi uterque; POAG, primary open-angle glaucoma; WHO, World Health Organization.

Table 5 Comparison of HVF test results between the moderate and advanced primary open-angle glaucoma patients

\begin{tabular}{llll}
\hline Variable & $\begin{array}{l}\text { Advanced } \\
\text { patients }\end{array}$ & $\begin{array}{l}\text { Moderate } \\
\text { group }\end{array}$ & P value \\
\hline Patients & 19 & 19 & \\
Male/female & $13 / 6$ & $9 / 10$ & 0.32 \\
MD mean (SD) & $-17.38(9.95)$ & $-6.69(5.38)$ & $<0.00$ I \\
PSD mean (SD) & $6.7 I(4.62)$ & $5.11(5.03)$ & $0.18^{*}$ \\
Age (years) & $53.89(16.5 \mathrm{I})$ & $52.68(14.30)$ & $0.8 \mathrm{I}$ \\
IOP & $36.46(12.40)$ & $23.03(6.95)$ & $<0.00$ I \\
CDR & $0.83(0.20)$ & $0.57(0.15)$ & $<0.00$ I \\
\hline
\end{tabular}

Notes: Of 164 charts reviewed, 69 eyes of 38 patients had reliable HVF results. Of the 38 patients, 19 had advanced and 19 had moderate POAG. There was no significant difference in male/female distribution, VA, or age. The MD of the advanced HVF group was significantly more negative than the moderate HVF group $(P<0.001)$. *The difference in PSD between groups was not initially significant due to two outliers with PSD $>15(P=0.18)$, but became significant after adjusting for these data points $(P<0.05)$ (see Figure 3).

Abbreviations: VA, visual acuity; IOP, intraocular pressure; CDR, cup-to-disk ratio; HVF, Humphrey visual field; MD, mean deviation; POAG, primary open-angle glaucoma; PSD, pattern standard deviation. old affected, to approximately $1 \%$ at 70 years, and well over 3\% at 75 years. The St Lucia and Barbados cohorts both showed a steady increase in age-associated prevalence. The major population study in Ghana reported that POAG affects $7.7 \%$ and $8.5 \%$ of people 30 and 40 years of age, respectively. ${ }^{3}$ These figures are expected to increase as life expectancy increases in Ghana and will pose an increasing burden on eye care services in the outpatient setting. ${ }^{17}$

Elevated IOP is currently thought of as a major risk factor and no longer the defining characteristic of POAG. ${ }^{7}$ There is some evidence to show cyclical fluctuations in IOP occur during a 24-hour day. ${ }^{18}$ Results are conflicting as to whether these fluctuations, measured in terms of the standard deviation for multiple office measures, lead to progression of POAG. ${ }^{19,20}$ For this study IOP was recorded as the average of two consecutive measurements taken from the patient chart prior to medical therapy. We feel that the accuracy of our definitions and findings were increased using this method.

In a previous case-control study in Ghana, glaucoma progression was determined to be most strongly correlated with IOP $>30 \mathrm{mmHg}$ and a family history. ${ }^{5}$ Our study supports this conclusion, however we can only draw associations from our findings without any temporal relationship between the two POAG groups. We further show a strong association between HVF MD values as independent predictors of POAG progression and pre-treatment IOP. MD greater than $-12 \mathrm{~dB}$ is strongly associated with POAG stage and elevated IOP in this study.

Our study identified a significant difference in optic nerve asymmetry between the moderate and advanced glaucoma groups $(P=0.001)$ which indicates more severe unilateral optic neuropathy than bilateral optic neuropathy in these patients. It is not clear if this corresponds with our VA results which showed a significant difference in visual acuity between patient groups in OD only. What is clear is a high number of patients in the advanced POAG group met criteria for WHO blindness (VA > 20/200) which suggests the advanced POAG had affected central visual acuity more frequently in this patient group. Bilateral VA $>20 / 200$ was uncommon. This appears to suggest VA is a poor predictor of early POAG progression, but a better predictor of late optic nerve damage. Wilson et al supported this finding by showing evidence that the deterioration of visual acuity correlated with faster progression of POAG in black populations. ${ }^{21}$ In comparisons of VA between groups, there was a discrepancy in OS when compared as $\operatorname{logMAR}$ integers. This is accounted for by the fact that a higher number of patients 
A

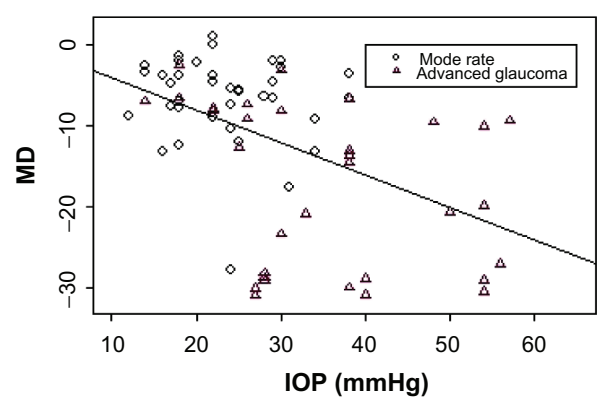

C

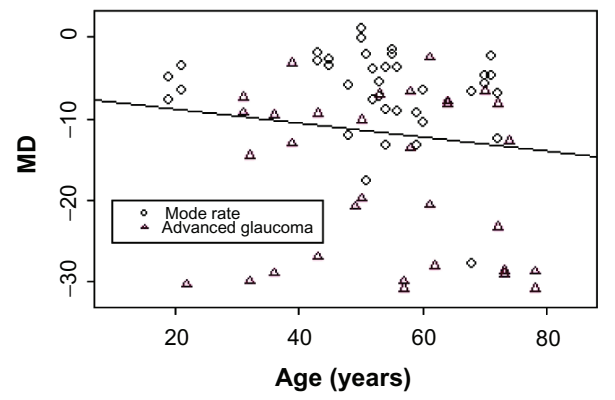

B

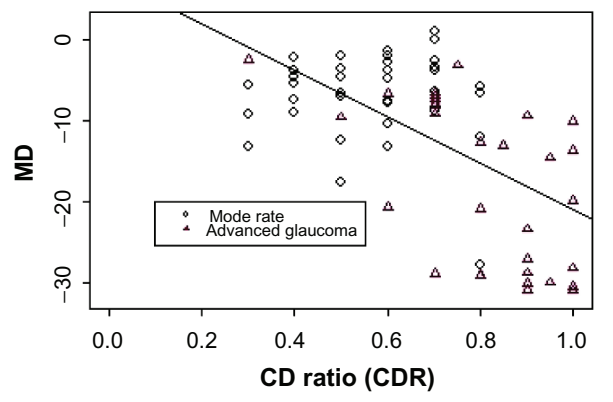

D

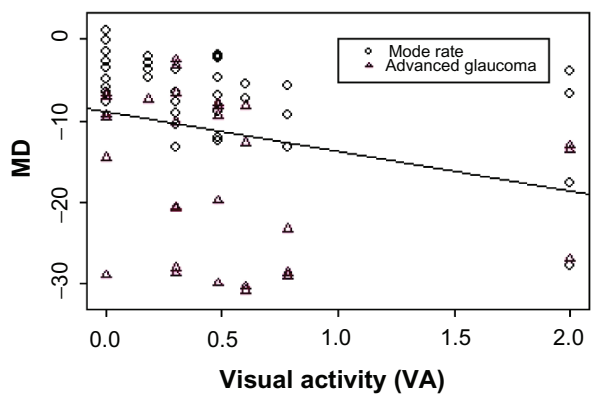

Figure 2 Comparisons of intraocular pressure, cup-to-disk ratio, age, and visual acuity as a function of visual field test mean deviation (MD) values for moderate and advanced primary open angle glaucoma groups.

Notes: As shown in A-D, MD values are negatively correlated with IOP (P, 0.0I). MD values are also negatively correlated with CDR (P, $0.00 \mathrm{I})$. MD values weakly, but statistically significantly correlate with BCVA (logMAR) $(P<0.01)$. MD values correlate best with IOP $(P<0.00 \mathrm{I})$ and $C D R(P<0.0 \mathrm{I})$, but not with increasing age $(P=0.49)$. (A) IOP as a function of MD. (B) CDR vs MD. (C) Age vs MD. (D) VA vs MD.

Abbreviations: VA, visual acuity; CDR, cup-to-disk ratio; MD, mean deviation; IOP, intraocular pressure.

A

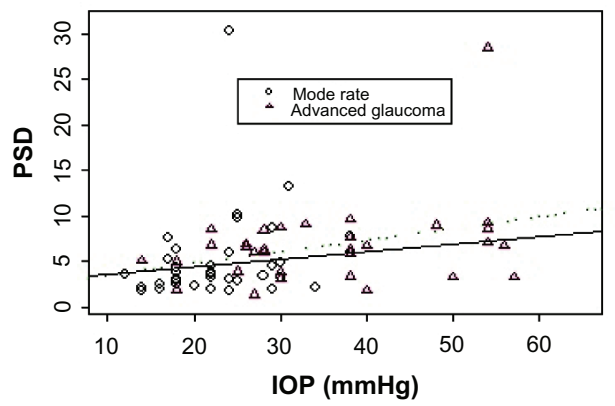

C

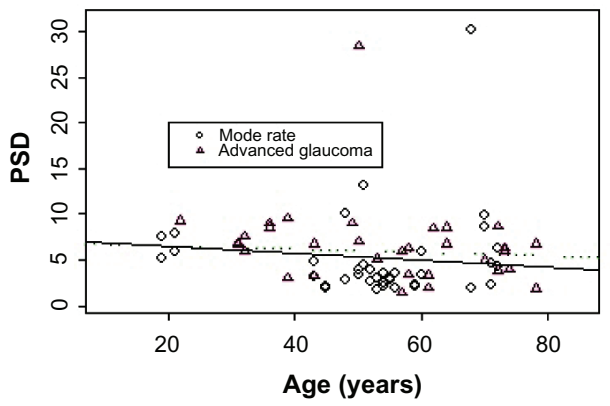

B

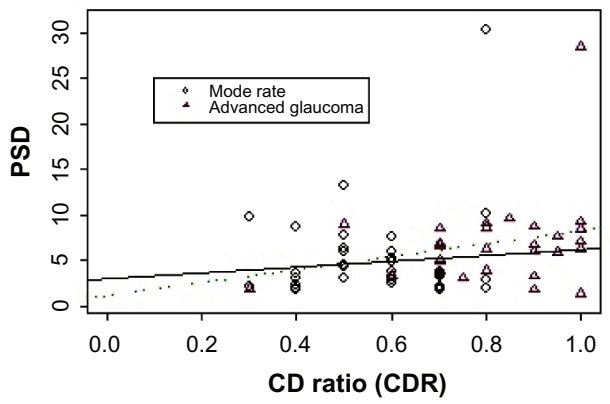

D

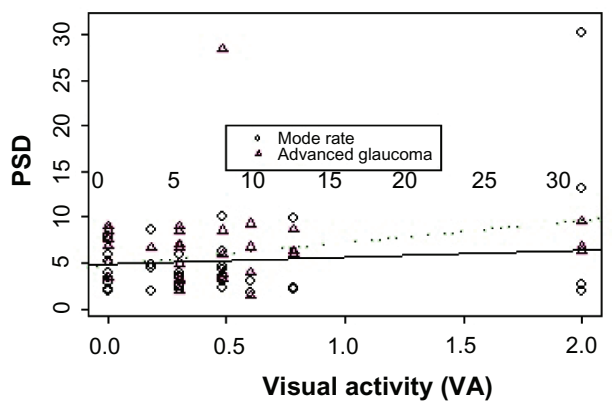

Figure 3 Comparisons of intraocular pressure, cup-to-disk ratio, age, and visual acuity as a function of visual field test pattern standard deviation (PSD) values for moderate and advanced primary open angle glaucoma groups.

Notes: Two regression lines are shown to account for differences in PSD with and without two outliers. The solid dark line excludes two outliers with PSD $>$ I5. The dotted lines are for all subjects. (A) There was a significant correlation between IOP and PSD with or without including the outliers (both $P \leq 0.0125)$. (B) The correlation between CDR and PSD was significant with all subjects $(P=0.0186)$, but fell short of significance when excluding the outliers $(P=0.0766)$. (C) There was no significant correlation between PSD and age $(P=0.653 \mathrm{I}$ for all subjects; $P=0.1027$ without the outliers). (D) The correlation between VA and PSD was also significant with or without the outliers (both $P \leq 0.023)$.

Abbreviations: VA, visual acuity; CDR, cup-to-disk ratio; IOP, intraocular pressure; PSD, pattern standard deviation. 
with HM, LP, or NLP in OS were omitted as these visual acuities do not correspond to a specific logMAR integer. Consequently the $\log$ MAR VA analysis result with missing information was less significant than the analysis using ordinal data.

Central corneal thickness is associated with IOP and progression of POAG. ${ }^{22,23}$ Corneas thinner than $520 \mu \mathrm{m}$ display lower IOPs, whereas thicker corneas display higher IOPs. Pachymetry testing was not available at this clinic. Reduced central cornea thickness has been reported in Black populations. ${ }^{24}$ Pachymetry testing may have led to a further elevation of IOP results in this study.

We are the first to report in a West African population a strong positive predictive ratio between estimation of CDR and HVF MD and PSD results. A significant implication of this finding is that it encourages clinicians in Ghana to utilize automated perimetry testing as part of the standard glaucoma work up and provides a measure of assurance to clinicians unable to obtain a clear view of the optic nerve head due to a variety of factors or those treating POAG suspects who present with normal IOP. Although CDR is less diagnostic in comparison to newer imaging modalities such as ocular coherence tomography (OCT) and Heidelberg retinal tomography (HRT), it still has a role in identifying patients at risk for POAG progression in resource poor clinical settings, especially when combined with HVF automated perimetry and the identification of a clear optic nerve asymmetry. Obstacles to effective HVF testing include patient factors such as poor concentration, dementia, and other mental and physical problems as well as clinician factors such as the high cost of an automated threshold perimeter, time to achieve useful results, and training technicians to operate the device and gather reliable data.

A limitation of this study was incomplete records for HVF MD and PSD. Patient charts showed that more than $80 \%$ of patients had HVF satisfactorily completed. HVF reports are given to the patient and copies were not consistently made for the clinician records. All available results were reliable and unreliable results were repeated at the clinic's expense. We acknowledge this reduced the power of our findings.

\section{Conclusion}

Comparisons of two POAG glaucoma groups at different stages of disease severity were undertaken to identify associations between clinical findings. Significant differences were found between IOP, CDR, MD and PSD values. HVF is predictive of pretreated IOP, CDR, and severity of POAG and it is strongly encouraged as part of the standard glaucoma work up in all Ghanaian patients.

\section{Acknowledgments}

An International Health Research Scholarship from Boston University School of Medicine, Boston, MA, 2009 and The Massachusetts Lions Eye Research Fund to Boston University provided funding/support. Design of the study (AF, MG, HG); conduct of the study (AF, MG); collection of data (AF, MG); management of data (AF, MG); analysis of data (AF, LD); interpretation of data (AF, MG, LD, $\mathrm{HG}$ ); preparation, review and approval of the manuscript (AF, MG, LD, HG). This manuscript has not previously been published and is an original manuscript. The corresponding author had full access to all the data in the study and takes responsibility for the integrity and accuracy of the data and its analysis as well as the decision to submit for publication. This retrospective study is in accordance with Health Insurance Portability and Accountability Act regulations and followed the tenets of the Declaration of Helsinki and received an exemption from an institutional review board (IRB) review in accordance with the following IRB criteria: B1.

\section{Disclosure}

There are no conflicts of interest associated with this work. The authors have no proprietary or commercial interest in any materials discussed in this article.

\section{References}

1. Leske MC. The epidemiology of open-angle glaucoma: a review. Am J Epidemiol. 1983;118(2):166-191.

2. Quigley HA. Number of people with glaucoma worldwide. $\mathrm{Br} J$ Ophthalmol. 1996;80(5):389-393.

3. Ntim-Amponsah CT, Amoaku WM, Ofosu-Amaah S, et al. Prevalence of glaucoma in an African population. Eye (Lond). 2004;18(5): 491-497.

4. Friedman DS, Wolfs RC, O'Colmain BJ, et al. Prevalence of openangle glaucoma among adults in the United States. Arch Ophthalmol. 2004;122(4):532-538.

5. Ntim-Amponsah CT, Amoaku WM, Ewusi RK, et al. Evaluation of risk factors for advanced glaucoma in Ghanaian patients. Eye (Lond). 2005; 19:528-534.

6. Herndon LW, Challa P, Ababio-Danso B, et al. Survey of glaucoma in an eye clinic in Ghana, West Africa. J Glaucoma. 2002;11(5):421-425.

7. Gyasi ME, Amoaku MK, Debrah OA, Awini EA, Abugri P. Outcome of trabeculectomies without adjunctive antimetabolites. Ghana Med J. 2006; $40(2): 39-44$

8. Hagan M. 2004 National Eye Care Report. Accra: Ghana Health Service Eye Care; 2005:3-5.

9. Foster P, Buhrmann R, Quigley H, Johnson GJ. The definition and classification of glaucoma in prevalence surveys. $\mathrm{Br} J$ Ophthalmol. 2002;86(2):238-242.

10. Racette L, Wilson MR, Zangwill LM, Weinreb RN, Sample PA. Primary open angle glaucoma in blacks: a review. Surv Ophthalmol. 2003;48(3): 295-313. 
11. Mason RP, Kosoko O, Wilson MR, et al. National survey of the prevalence and risk factors of glaucoma in St. Lucia, West Indies. Part 1. Prevalence findings. Ophthalmology. 1989;96(9):1363-1368.

12. Leske MC, Connell AM, Schachat AP, Hyman L. The Barbados eye study. Prevalence of open angle glaucoma. Arch Ophthalmol. 1994; 112(6):821-829.

13. Buhrmann RR, Quigley HA, Barron Y, West SK, Oliva MS, Mmbaga BB. Prevalence of glaucoma in a rural East African population. Invest Ophthalmol Vis Sci. 2000;41(1):40-48.

14. Rotchford AP, Johnson GJ. Glaucoma in Zulus: a population-based cross-sectional survey in a rural district in South Africa. Arch Ophthalmol. 2002;120(4):471-478.

15. Mukesh BN, McCarty CA, Rait JL, Taylor HR. Five-year incidence of open-angle glaucoma: the visual impairment project. Ophthalmology. 2002;109(6):1047-1051.

16. Leske MC, Heijl A, Hyman L, et al. Predictors of long-term progression in the early manifest glaucoma trial. Ophthalmology. 2007;114(11): 1965-1972.

17. Sory E, Nyonator F. The Health Sector in Ghana, Facts and Figures 2009. Accra: Ghana Health Service; 2009.

18. Fogagnolo P, Orzalesi N, Ferreras A, Rossetti L. The circadian curve of intraocular pressure: can we estimate its characteristics during office hours? Invest Ophthal Vis Sci. 2009;50(5):2209-2215.
19. Bengtsson B, Leske MC, Hyman L Heijl A; Early Manifest Glaucoma Trial Group. Fluctuation of intraocular pressure and glaucoma progression in the early manifest glaucoma trial. Ophthalmology. 2007;114(2): 205-209.

20. Lee PP, Walt JW, Rosenblatt LC, Siegartel LR, Stern LS; Glaucoma Care Study Group. Association between intraocular pressure variation and glaucoma progression: data from a United States chart review. Am J Ophthalmol. 2007;144(6):901-907.

21. Wilson R, Richardson TM, Hertzmark E, Grant WM. Race as a risk factor for progressive glaucomatous damage. Ann Ophthalmol. 1985; 17(10):653-659.

22. Herndon LW, Weizer JS, Stinnett SS. Central corneal thickness as a risk factor for advanced glaucoma damage. Arch Ophthalmol. 2004; 122(1):17-21.

23. Brandt JD, Beiser JA, Kass MA, Gordon MO. Central corneal thickness in the Ocular Hypertension Treatment Study (OHTS). Ophthalmology. 2001;108(10):1779-1788.

24. La Rosa FA, Gross RL, Orengo-Nania S. Central corneal thickness of Caucasians and African Americans in glaucomatous and nonglaucomatous populations. Arch Ophthalmol. 2001;119(1):23-27.

\section{Clinical Ophthalmology}

\section{Publish your work in this journal}

Clinical Ophthalmology is an international, peer-reviewed journal covering all subspecialties within ophthalmology. Key topics include: Optometry; Visual science; Pharmacology and drug therapy in eye diseases; Basic Sciences; Primary and Secondary eye care; Patient Safety and Quality of Care Improvements. This journal is indexed on

\section{Dovepress}

PubMed Central and CAS, and is the official journal of The Society of Clinical Ophthalmology (SCO). The manuscript management system is completely online and includes a very quick and fair peer-review system, which is all easy to use. Visit http://www.dovepress.com/ testimonials.php to read real quotes from published authors. 